pital: est-il possible d'étendre les terres arables, qui manquent partout cruellement aux Pays-Bas, en diminuant au maximum le fumier (donc le bétail et les prairies).

A tous points de vue le Polder du Nord-Est constitue donc un véritable creuset, une région-pilote de l'agriculture néerlandaise ${ }^{1}$.

1 Les documents qui nous ont servi pour cet article sont, outre les explications des ingénieurs agricoles néerlandais qui nous ont conduits à travers le Polder du N. E., la brochure „Essai sur le Polder du Nord-Est", éditée à Kampen, au printemps 1954, par la Direction des Travaux du Polder N.E.; une autre, plus restreinte, éditée par le même service, sous le même titre, sans date; la brochure "Les Pays-Bas", par Dr Tosseram, Amsterdam, 1953; la brochure " Dutch Agriculture : Facts", publiée en 1953 par le service d'information du ministère de l'agriculture.

R. L.

\title{
NEUE SCHULKARTE DES KANTONS SCHAFFHAUSEN
}

Die Erziehungsdirektion des Kantons Schaffhausen hat für ihre Schulen eine neue Handkarte, bearbeitet von Prof. Dr. h. c. EdUARD IMHof, herausgegeben. Es ist eine Kurvenkarte mit Reliefmalerei („Schweizer Manier“) im Maßstab 1:75000 und mit der Aequidistanz 20 Meter. (Das sind die Minimalworte, mit denen das kartographische Werk in den Kartotheken registriert werden wird.)

An eine Karte vom modernen Typus der vorliegenden werden hohe Forderungen gestellt. Sie soll generalisieren: das nicht notwendige Detail der Klarheit des Gesamtbildes opfern. Sie soll aber im Generalisieren landschaftskundlich gewissenhaft individualisieren: aus dem Gesamtbilde Landschaftsindividuen herausarbeiten. Dieses zeichnerische Modellieren von Landschaftskörpern, so $\mathrm{da}$ ß ihre organische Einheit sichtbar wird - dieses Verdeuten von Wesenheit, Erfassen landschaftlicher Persönlichkeit, ist im Prinzip Hodlerisches Gestalten. Der künstlerische Kartograph mag die Anfeindung durch den nur-technischen Darsteller erleben - er allein aber ist der wirklich schöpferische und wahrheitsgemäße Nachbildner der Landschaft. Der gelegentliche Vorwurf, daß solche Darstellung "nicht wissenschaftlich" sei, grenzt an die alte verheerende Auffassung, daß der Wissenschaft die Vernachlässigung der Form zuzubilligen sei oder gar zur Ehre gereiche. „Kunst und Wissenschaft dürfen in der Kartographie nicht gegeneinander ausgespielt werden. Sie streben gemeinsam nach Formentreue, graphischer Klarheit und leichter Lesbarkeit der Karten." (E. IMHOF in "Gelände und Karte“.)

Jede neue Übersichtskarte entsteht aus den Grundlagen bisheriger topographischer Aufnahmen. Für die neue Schaffhauser Schulkarte bestanden ungewöhnliche Schwierigkeiten. Die gegliederten Umrißverhältnisse des Kantons erforderten einen starken Einbezug von Nachbargebieten. Außerdem erleichtert die zeichnerische Mitberücksichtigung von Nachbarlandschaften die landschaftskundliche Auswertung eines Karteninhaltes. Diese Überlegungen bestimmen die Wahl des Umrisses und des Maßstabes einer Übersichtskarte.

Als Grundlage sind die schweizerischen Siegfriedblätter veraltet, sowohl inhaltlich als auch in ibrer Genauigkeit. Das Gleiche gilt für die badischen Meßtischblätter 1:25000. Für das Schaffhausergebiet lagen noch keine Blätter der neuen „Landeskarte der Schweiz“ vor. „Es war daher unerläßlich, durch lokale Auskünfte, durch Feldbegehungen, durch Meliorationspläne, durch Luftbilder (Flugphotos) usw. das mangelhafte Grundlagematerial zu überprüfen und zu ergänzen “ (Imhof). Die Höhen mußten auf den „neuen Horizont“ (Pierre de Niton 373,6 Meter) transformiert werden. Der Horizontunterschied von 2 Metern zwischen den deutschen und den schweizerischen Karten war einzurechnen. In einzelnen Gemeinden standen neue Grundbuchpläne zur Verfügung. Aus dem Splittermaterial solcher Grundlagen mußte ein einheitliches und modernes Kartenbild geschaffen werden. Als Herausgeberin zeichnet eine Schulbehörde. Das Werk mußte also dem Anspruch der Vorbildlichkeit genügen. In der kartographischen Vorbildlichkeit war aber zugleich jene augenfällige Einfachheit des Anschaulichen vorausgesetzt, welche das Verständnis und forschungsfreudige Interesse des jungen Schülers weckt.

War es möglich aus den genannten Voraussetzungen solche Forderung zu erfüllen? Der Gipfel des Zieles stand hoch und hindernisbelagert über der tiefen Ausgangsbasis. Er ist vollkommen bestiegen worden.

In der Arbeit des Zusammentragens von Werten, Namen und heimatkundlichen Einzelheiten verschiedenster Art, wurde der Kartograph unterstützt von Kantonsgeometern, Vorstehern von Meliorations- und Vermessungsämtern, von Lehrern und Heimatkennern. Kommt das gesammelte und gesichtete Material in die Obhut des landschaftskundlich geschulten Kartographen, so entsteht nun nicht nur ein Bild der Summierung dieser Details in kartenzeichnerisch mehr oder weniger persönlicher Darstellung. Es folgt eine Gestaltungsarbeit, welche das Gelände in morphogenetischer Aufmerksamkeit beurteilt und die Landschaft nach ihren anatomischen Prinzipien modelliert. Die Realität des Details wird durchaus erfaßt, aber sie wird eingewertet in die morphologische Konzeption und drängt sich nicht in einer scheinbaren Wissenschaftlichkeit vor auf Kosten der wahrheitsgemäßeren Erfassung des organischen Gesamtbildes. So wächst ein kartographisches Landschaftsbild, das technisch treu und nach künstlerischer Zielsetzung gestaltet ist. Nicht allein zuverlässige 
Wiedergabe der Landschaft will es sein, sondern zugleich ihre getreue Interpretation. Damit überhaupt erst ist die Karte nicht nur ein technisches, sondern ein wissenschaftliches Phänomen.

So läßt man mit Genuß den Blick über dieses Kartenwerk wandern von Landschaftsbild zu Landschaftsbild. Hart springen im Norden die Randenhöhen aus der Karte. Der Gegensatz ihres jurassischen Charakters zeichnet sich prägnant von den weicher modellierten Tafeln und Rücken der Molasseberge südlich des Rheines ab. In betontem Licht-Schattenkontrast ragen die Vulkanruinen des Hegau aus dem flachen Umland. Ihr fremdartig-eigenwilliges Landschaftsmotiv lockt zum Lehrgespräch. Im Nordwesten beginnt das Fiederwerk der Schwarzwaldtäler. Diesem Landschaftsglied stark fluvialer Prägung stehen im Osten des Kartenraumes die breit und parallel hereinziehenden Wannen der glazialmodellierten Täler der ehemaligen Rhein- und Thurgletscherarme gegenüber. Thur- und Stammheimertal sind schorfig ummalt von Moränenlandschaften. Endmoränenbogen und Zungenbecken drängen selbst dem Schüler Erklärungsversuche auf die Zunge. Die Karte erfaßt sogar noch mit Kurven und mit feinster Reliefmalerei die Miniaturhügelwelt von Drumlinlandschaften bei Hettingen und westlich von Frauenfeld. Leichte Schattenstreifen modellieren die Ränder von Schotterterrassen. Und immer wieder fällt der Blick in die Mitte der Karte, wo Hauptstadt und Rheinlauf das Auge fesseln. Da windet sich, cañongleich in die Schottertafel eingeschnitten - die Karte erreicht hier ihre feinste Landschaftsfühlung - der einzigartige Stromlauf Rheinfall-Rheinau: der sterbende Strom. Noch allerdings hat die Karte die Sperrungen zu seiner Drosselung nicht in ihr Bild aufgenommen. Vom Schaffhauser Rheinknie springt ein Fächer von Seitentälern in den Randen ein. Die wachsende Stadt streckt ihre Häuserarme in diese Täler vor. Den Siedlungen wurde in der Karte große Aufmerksamkeit geschenkt. Sie springen stark aus dem Kartenbild ohne die Reliefmodellierung zu beeinträchtigen. Rasch sind Zusammenhänge erkennbar. Kleindörfer, vor allem Weiler und Einzelhöfe, sind in die höckerigen Moränenlandschaften eingetupft. Große Haufen- und Straßendörfer reihen sich den Rändern der Talebenen, Schotterflächen und Zungenbecken entlang. Sie mieden nicht allein die einstigen Sümpfe, die man noch in der Leere weiter Talfächen ahnt; sie nützen zwiefachen Wirtschaftsraum, haben die Rebberge hinter sich und vor sich die Felderflur der Ebene. Der Maßstab 1:75000 erlaubt nicht mehr die flächentreue Wiedergabe von Gebäuden. Sie liegen unter der Grenze des graphisch Darstellbaren. Eine „technische“ Ausführung der Karte kommt also gar nicht mehr in Frage. Fs kann lediglich noch auf Treue des Grundrisses der Siedlung geachtet werden. So wird der Kartograph zum Siedlungsgeographen, der dem Landschaftsbild der Karte den Siedlungstypus korrekt vermittelt.

In der Farbenmalerei liegt die eigentliche Darstellungskunst. Wie hart standen sich einst in den Reliefkarten die Farbtöne gegenüber, lediglich kontrastsüchtig und ohne morphologische Verantwortung. Die Farbtönung der Höhenstufen bewegt sich in der vorliegenden Karte von Graugrün steigend zu Gelb. In diese Farben sind die Waldfächen durch feinen grünen Punktraster eingetragen, daß die Wälder in der Karte sich als zarter Schleier über Ebenen und Berge legen, ohne auch die feingliedrigste Hügelmodellierung zu belasten. Stärker betont, in vertikaler Strichsignatur von weinroter Farbe, sind die Rebberge an die Hänge gelehnt - auf hellem Untergrund fast etwas zu stark hervortretend. Die Schattenlagen sind durch den üblichen Lichtwurf von Nordwesten gegeben. Und diese Schattenfarben nun, aus dem Grün der Ebenen zu dunkleren Tönen steigend, beherrschen stark den Bildwert einer Karte Hier fallen die Schatten in die Hänge ohne die übrigen Eintragungen zu ersticken. Die Kartenplastik ist deutlich und licht zugleich. Daß nun alle diese Farbtöne zum hervorragenden Kartenbild zusammenklingen, das fügt sich durch Willkür ebenso wenig wie die Melodie auf dem Notenblatt.

Gleichzeitig mit der Handkarte hat die Erziehungsdirektion des Kantons Schaffhausen eine Wandkarte im Maßstab 1:25000 herausgegeben. Durch die gemeinsame Gestaltung der zeichnerischen Originale für die beiden Karten war inhaltliche Übereinstimmung und zugleich wesentliche Einsparung gewährleistet.

Die beiden Karten sind begleitet von einem Heft „Erläuterungen zur Schulkarte des Kantons Schaffhausen 1954", worin die Mitwirkung von Mitgliedern der kantonalen Schulkartenkommission zu Worte kommt und verdankt wird. Erziehungsdirektor THEO WANNER stellt kurz das Werk vor. Professor IMHOF hält Rückblick über den inhaltlichen und Kartograph KURT FickeR über den technischen Werdegang. Professor Hermann Wanner zeigt die Sorgfalt, mit welcher die Orts- und Flurnamenschreibung in der Karte abgeklärt und überwacht wurde. Schulinspektor ERwin BüHLER bietet ein umfangreiches Verzeichnis der heimatkundlichen Einträge in der Karte. Darin liegt eine heimatkundliche Fundgrube, und man empfindet das Bedürfnis nach einer Gesamtdarstellung der Schaffhauser Heimatkunde, die dem Heft vielleicht nur darum nicht mitgegeben wurde, weil sie separat besteht. Denn der nicht besonders begabte Kartenleser wird erst durch das begleitende Wort zur Fülle der Entdeckungen geführt.

Die Reproduktion der Karten besorgte die Geographische Anstalt Kümmerly \& Frey in Bern. Es war ihr dabei Gelegenheit geboten, die modernsten Reproduktionsverfahren voll auszunützen. Zum ersten Male wurde eine Wandkarte durch Reproduktionsphotographie geschaffen. Es wurden eingehende Versuche gemacht, um die richtigste Methode zu finden zur Darstellung der Bodenbedeckung, welche sich klar ausprägt ohne das Spiel der Geländefarben zu beeinträchtigen.

Diese Schulkarte berechtigt nicht allein zu schaffhauserischem Stolz, sie ist ein Werk, das der schweizerischen Kartographie Ehre einlegt.

EMIL EGLI 